\title{
Structure and catalytic mechanism of the $E$. coli chemotaxis phosphatase CheZ
}

Rui Zhao, Edward J. Collins, Robert B. Bourret and Ruth E. Silversmith

Nature Structural Biology 9, 570-575 (2002).

The PDB accession code for the structure of $\mathrm{CheZ}-\mathrm{CheY}-\mathrm{BeF}_{3}{ }^{-}$was incorrectly reported in the 'Coordinates' section of this paper. The correct PDB accession code is $1 \mathrm{KMI}$. We apologize for any inconvenience this may have caused.

\section{Intrinsic metal binding by a spliceosomal RNA}

Saba Valadkhan and James L. Manley

Nature Structural Biology 9, 498-499 (2002).

A mistake occurred during the production of this News and Views report. The observation stated at the bottom of the second column on page 499 was incorrectly referenced to ref. 14 . The correct reference for this observation is ref. 12, and the correct sentence is printed as follows: “... the block to splicing due to thio substitution of either Sp oxygen in the domain V loop could not be rescued by $\mathrm{Mn}^{2+}$ (ref. 12).” We apologize for any inconvenience this may have caused. 